\title{
A 26-year-old competitive soccer player with syncope
}

\author{
Yasbanoo Moayedi MD, Ramanan Kumareswaran MD, Paul Dorian MD
}

A 26-year-old man experienced an unwitnessed syncopal event while brushing his teeth at night. He suffered trauma to his head and was found, unconscious on the floor, by his roommate. He did not have any signs of seizure, such as tongue-biting, incontinence or postictal confusion. He was admitted to hospital for further monitoring. His medical history was unremarkable. He was a competitive soccer player. The results of a physical examination, routine blood work and serum toxicology screening were unrevealing.

On the second day of admission, the patient was found unresponsive, and the telemetry tracing shown in Figure 1 was recorded.

\section{What is the rhythm shown on telemetry?}
a. Artifact
b. Atrial fibrillation
c. Ventricular fibrillation
d. Ventricular tachycardia

The answer is (c). The tracing depicts a telemetry strip (lead II) above an arterial pressure tracing. The fifth QRS complex is followed by an "early coupled" premature ventricular contraction (marked with an asterisk), which has resulted in a short-long-short sequence (marked as "s $1 \mathrm{~s}$ " in the figure) triggering ventricular fibrillation. The corresponding arterial tracing shows pulseless hypotension during ventricular fibrillation. The onset of the arrhythmia is not preceded by atrial activity, and the triggering beat is ventricular; these features, combined with the pulseless arterial tracing, make atrial fibrillation unlikely. This very fast and polymorphic arrhythmia is most consistent with ventricular fibrillation.

The patient was resuscitated according to advanced cardiac life support guidelines. ${ }^{1}$

\section{What diagnoses should be considered in this patient?}
a. Atherosclerotic heart disease
b. Hypertrophic cardiomyopathy
c. Primary electrical disease
d. Coronary artery anomaly
e. All of the above

Competing interests:

None declared.

This article has been peer reviewed.

The authors have obtained patient consent.

Correspondence to:

Paul Dorian,

dorianp@smh.ca

CMAJ 2015. DOI:10.1503 /cmaj.141140

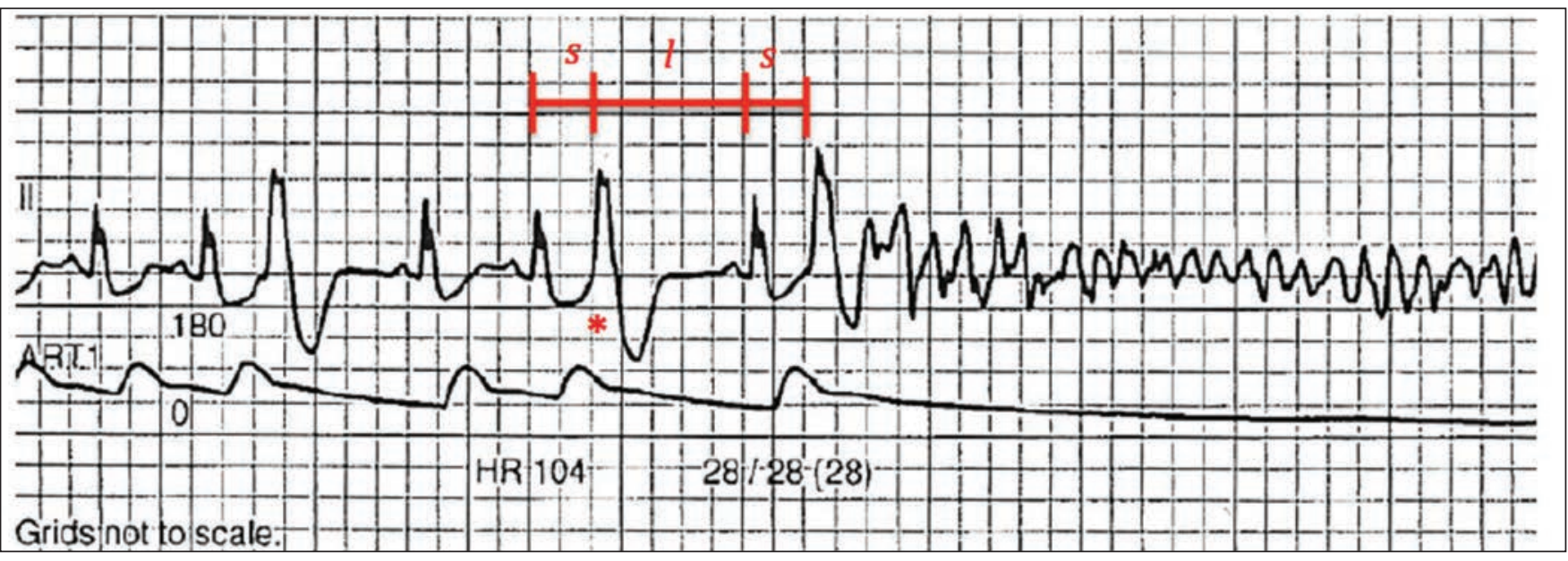

Figure 1: Telemetry tracing (lead II) above an arterial pressure tracing for a 26-year-old man who experienced a syncopal event. *"Early coupled" premature ventricular contraction. HR = heart rate, "s I s" = short-long-short sequence. 
fibrillation) in apparently healthy young individuals. An autopsy-based series showed that, among those 35 years of age or younger, primary electrical disease was the most common cause of death $(41.3 \%)$, followed by atherosclerotic disease $(23.2 \%)$ and hypertrophic cardiomyopathy $(12.8 \%) .^{2}$ An anomalous coronary artery was identified in less than $5 \%$ of these cases. ${ }^{2}$ In contrast, atherosclerotic heart disease was the most common cause of death among those over 35 years of age. ${ }^{2}$ In a Canadian study of unexplained cardiac arrest in the absence of overt cardiac structural abnormalities, a diagnosis was reached in $56 \%$ of patients; the diagnoses included long QT syndrome (23\%), catecholaminergic polymorphic ventricular tachycardia (23\%), arrhythmogenic ventricular cardiomyopathy (17\%), early repolarization (14\%), coronary spasm (11\%), Brugada syndrome (9\%) and myocarditis (1\%). ${ }^{3}$

Classifying these causes into mutually exclusive categories is difficult. A useful approach is to divide life-threatening arrhythmias into three major categories: structural, primary electrical and acute ischemic disease (Box 1)..$^{4-6}$ The underlying diseases may be congenital or acquired.

Some causes of sudden cardiac death do not fit within a single category. For example, arrhythmogenic right ventricular cardiomyopathy is a structural disorder, but it usually manifests with arrhythmia (i.e., electrical). Appendix 1 (available at www.cmaj.ca/lookup/suppl/doi:10.1503/cmaj .141140///DC1) illustrates the interplay between these categories.

\section{How should this patient be further evaluated?}

a. Detailed personal and family history, with comprehensive evaluation of an electrocardiogram (ECG)

b. Cardiac magnetic resonance imaging

c. Genetic testing for all congenital arrhythmias

d. Procainamide challenge to assess for Brugada syndrome
The answer is (a). Rather than adopting a "shotgun" or stereotyped approach to investigating the cause of "aborted" ventricular fibrillation in this patient, the clinician should obtain a detailed history, perform a physical examination and thoroughly assess the findings of 12-lead ECG with the heart in sinus rhythm., 5,6

The history should prompt details of previous episodes of syncope (particularly during exercise or with sleep), any trigger for syncope, presence of a prodrome, ischemic symptoms and family history of sudden cardiac death. As part of the family history, it is important to ask about clues to the long QT syndrome, such as drowning of a family member who was a good swimmer, an unexpected motor vehicle collision, congenital deafness and sudden infant death syndrome. It is also important to ask about a family history of hypertrophic cardiomyopathy and muscular dystrophy. A family history of sudden cardiac death at a young age may be a clue to a heritable heart rhythm disorder. The medication history should identify use of prescription, nonprescription and illicit drugs. ${ }^{5,6}$

The physical examination should focus on signs of potential structural causes of arrhythmia, including hypertrophic cardiomyopathy and valvular abnormalities. ${ }^{4}$

The ECG while in sinus rhythm should be carefully assessed for the following features: ${ }^{4-6}$

- ST-segment changes or findings of prior myocardial infarction (q waves)

- Duration of QT interval (long or short) and T-wave morphology

- Signs of Brugada syndrome, with characteristic right-bundle type QRS morphology and ST-segment elevation in leads $\mathrm{V}_{1}$ and $\mathrm{V}_{2}$

- Left ventricular hypertrophy with associated repolarization changes

- Signs of Wolff-Parkinson-White syndrome, with short PR segment and slurred QRS upstroke (delta wave)

- Signs of arrhythmogenic right ventricular cardiomyopathy with epsilon waves and $\mathrm{T}$-wave abnormalities in the right precordial leads $\left(\mathrm{V}_{1}-\mathrm{V}_{3}\right)$

\section{Box 1: Causes of life-threatening arrhythmia}

Structural heart disease: Any structural abnormality involving the coronary arteries, valves or ventricles that is suspected to be the main cause of sudden cardiac death. Congenital forms include hypertrophic cardiomyopathy, arrhythmogenic right ventricular cardiomyopathy, left ventricular noncompaction cardiomyopathy, coronary anomaly, and repaired or unrepaired congenital heart disease. Acquired forms include viral myocarditis, sarcoidosis and dilated cardiomyopathy. ${ }^{4}$ Diagnostic tools, including imaging, do not always identify structural abnormalities. ${ }^{4-6}$

Primary electrical disorders: Congenital forms include long QT syndrome, short QT syndrome, Brugada syndrome, catecholaminergic polymorphic ventricular tachycardia, Wolff-Parkinson-White syndrome and idiopathic ventricular fibrillation. Acquired causes include drug-induced QT prolongation, hypokalemia, hypomagnesemia, electrical shock and commotio cordis. ${ }^{5}$

Acute ischemic causes: Include atherosclerotic disease, coronary artery embolism, coronary vasospasm, coronary artery dissection and coronary vasculitis. ${ }^{5}$ 
The sequence and nature of tests thereafter are contingent on the patient's pretest probability for a particular cause for sudden cardiac death. For example, for patients with findings of ischemia (e.g., clinical symptoms, ischemic ECG abnormalities or elevated troponin), further testing with coronary angiography to assess for coronary anomaly and coronary artery disease is recommended. ${ }^{4}$

Transthoracic echocardiography is relatively sensitive for many structural abnormalities. However, in certain instances, especially right ventricular disease, echocardiography can miss subtle abnormalities and further imaging may be indicated. ${ }^{6,7}$ Further testing is discretionary and should involve consultation with an electrophysiologist.

\section{On the basis of postdefibrillation ECG, what is the most likely diagnosis?}
a. Anterior myocardial infarction
b. Incomplete right bundle branch block
c. Brugada syndrome
d. Early repolarization

The answer is (c). The ECG, obtained after cardiac defibrillation (Figure 2), showed a right bundle branch block-type pattern and characteristic 2-mm coved ST-segment elevation in right precordial leads $\mathrm{V}_{1}$ and $\mathrm{V}_{2}$. This ECG finding is classic for the "Brugada type 1 pattern."

\section{Discussion}

Brugada syndrome is associated with a sodiumchannel dysfunction and is most prevalent in middle-aged Asian and Southeast Asian men. ${ }^{6}$ The most concerning pattern on ECG is type 1, associated with the highest risk of sudden death. It may be present intermittently or continuously or may be unmasked with provocative drug testing (e.g., infusion of a sodium-channel blocker) ${ }^{6,7}$ Brugada syndrome is a rare cause of sudden cardiac death, with a prevalence of less than $5 \%$ of cases. ${ }^{3,7}$

Clinical manifestations of Brugada syndrome include syncope, nocturnal agonal breathing, palpitations, ventricular fibrillation and sudden cardiac death. These symptoms commonly occur during sleep or can be exacerbated by fever and sodium-channel blocker drugs. ${ }^{6,7}$

Brugada syndrome is associated with an autosomal dominant pattern of transmission with variable penetrance. Mutations in the sodiumchannel gene (SCN5A) may be found; however, fewer than $30 \%$ of patients with the Brugada syndrome phenotype have a positive result on genetic testing. Neither a family history of sudden cardiac death nor the presence of a gene mutation has proven helpful in stratifying risk. ${ }^{6}$

Patients with Brugada syndrome are counselled to avoid drugs that block sodium channels, including psychotropic drugs such as amitriptyline, lithium and loxapine (a full list of such drugs is available at www.brugadadrugs.org).$^{6-8}$ Other precipitants, such as excessive alcohol intake,

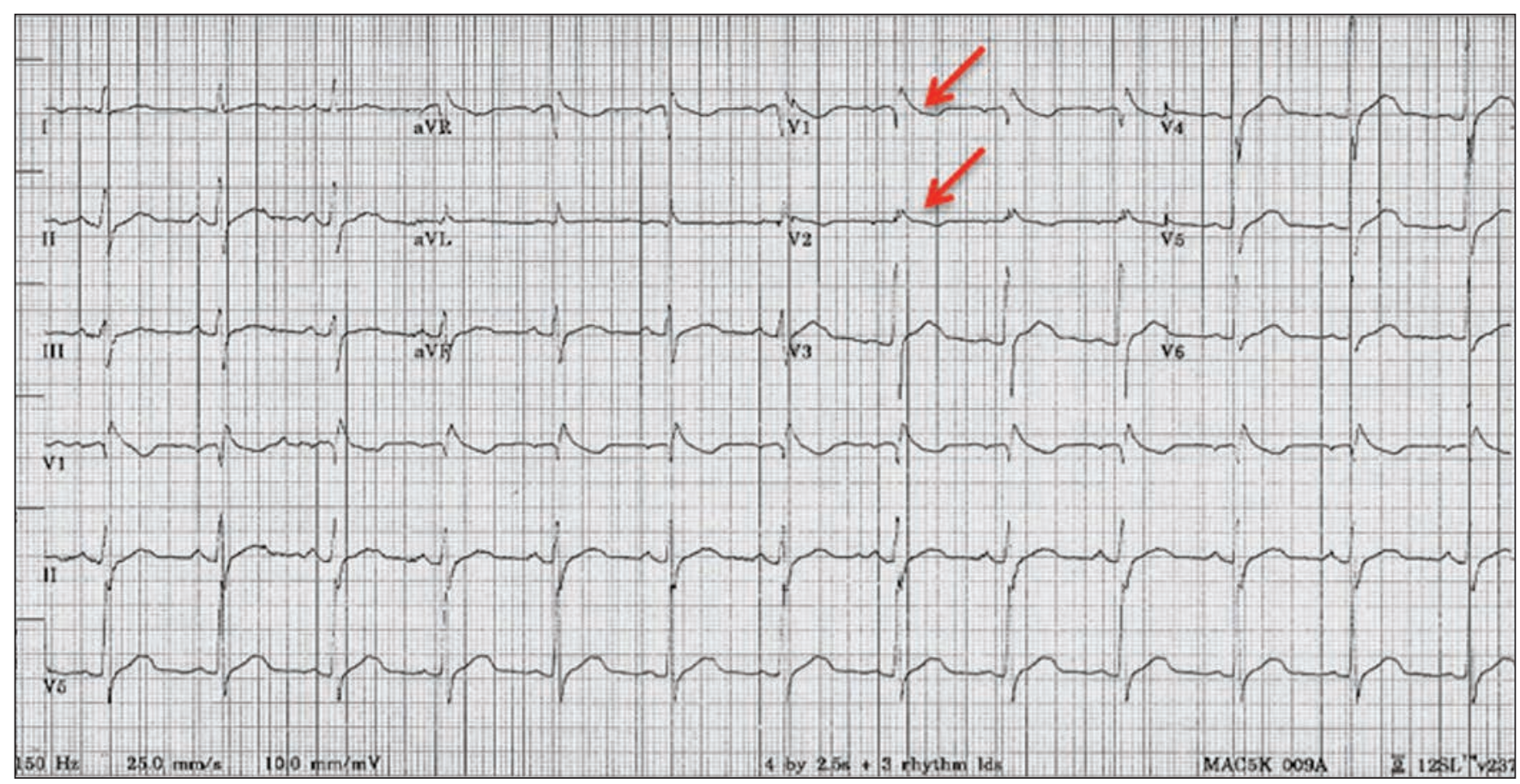

Figure 2: Twelve-lead electrocardiogram showing incomplete right bundle branch block with coved ST elevation in precordial leads $\mathrm{V}_{1}$ and $\mathrm{V}_{2}$ (arrows). 
cocaine and hot tub use, should also be avoided. Immediate antipyretic therapy for fever is extremely important.

Implantation of an internal cardioverterdefibrillator is recommended for survivors of cardiac arrest but not for primary prevention. ${ }^{6}$ Because Brugada syndrome is an autosomal dominant familial condition, current guidelines recommend testing for all first-degree relatives (parents, children and siblings) with a clinical assessment and 12-lead ECG. ${ }^{5}$

\section{Cardiovascular screening in young athletes}

The role of screening young people, particularly athletes, for risk factors for sudden cardiac death is complex and controversial. ${ }^{9,10}$ The American Heart Association has recommended preparticipation cardiovascular screening, along with a history and physical examination, to guide further testing. ${ }^{9}$ Conversely, the European Society of Cardiology includes preparticipation ECG as part of routine screening. ${ }^{11}$ Some studies (e.g., that of Corrado and associates ${ }^{12}$ ) have suggested that screening programs may lead to a reduction in sports-related sudden cardiac death; however, subsequent studies by other researchers, including Sharma and colleagues, ${ }^{10}$ have not replicated these findings.

Many factors limit the practicality and costefficiency of widespread ECG screening, including the number of athletes, the low prevalence of disease and the high number of false positives, which far exceed true positives.

Importantly, such screening would exclude nonregistered athletes and non-athletes; would apply primarily to rhythm disorders that are related to or precipitated by exercise; and would assume, without good evidence, that restricting competitive sport among those believed to be at risk will reduce the incidence of sudden death.

In light of the controversies and complexities associated with preparticipation screening, clinicians evaluating such patients should be highly experienced and should use informed judgment to further stratify, according to risk, patients who might benefit from additional testing.

In this particular case, there is no good reason to believe that screening would have had any benefit, as the ECG findings in Brugada syndrome can be intermittently normal, and even if Brugada type 1 is discovered on ECG screening in an asymptomatic patient, prophylaxis is not indicated. If this patient had been counselled against participating in or barred from competitive sport (for which there would have been no evidence of benefit), there could be no expectation that arrhythmias would be prevented from occurring at rest, as is usual with this condition.

\section{References}

1. Field JM, Hazinski MF, Sayre MR, et al. Part 1: executive summary: 2010 American Heart Association guidelines for cardiopulmonary resuscitation and emergency cardiovascular care. Circulation 2010;122(18 Suppl 3):S640-56.

2. Eckart RE, Shry EA, Burke AP, et al. Sudden death in young adults: an autopsy-based series of a population undergoing active surveillance. J Am Coll Cardiol 2011;58:1254-61.

3. Krahn AD, Healey JS, Chauhan V, et al. Systematic assessment of patients with unexplained cardiac arrest: Cardiac Arrest Survivors with Preserved Ejection Fraction Registry (CASPER). Circulation 2009;120:278-85.

4. Pelto H, Owens D, Drezner J. Electrographic findings suggestive of cardiomyopathy: what to look for and what to do next. Curr Sports Med Rep 2013;2:77-85.

5. Priori SG, Wilde AA, Horie M, et al. HRS/EHRA/APHRS expert consensus statement on the diagnosis and management of patients with inherited primary arrhythmia syndromes. Heart Rhythm 2013;10:1932-63.

6. Krahn AD, Sanatani S, Gardner MJ, et al. Inherited heart rhythm disease: negotiating the minefield for the practicing cardiologist. Can J Cardiol 2013;29:122-5.

7. Modi S, Krahn AD. Contemporary reviews in cardiovascular medicine: sudden cardiac arrest without overt heart disease. Circulation 2011;123:2994-3008

8. Postema PG, Wolpert C, Amin AS, et al. Drugs and Brugada syndrome patients: review of the literature, recommendations and an up-to-date website (www.brugadadrugs.org). Heart Rhythm 2009;6:1335-41.

9. Maron BJ, Thompson PD, Ackerman MJ, et al. Recommendations and considerations related to preparticipation screening for cardiovascular abnormalities in competitive athletes: 2007 update: a scientific statement from the American Heart Association Council on Nutrition, Physical Activity, and Metabolism. Circulation 2007; 115:1643-55.

10. Sharma S, Merghani A, Gati S. Cardiac screening of young athletes prior to participation in sports: difficulties in detecting the fatally flawed among the fabulously fit. JAMA Intern Med 2015;175:125-7.

11. Corrado D, Pelliccia A, Bjørnstad HH, et al. Cardiovascular pre-participation screening of young competitive athletes for prevention of sudden death: proposal for a common European protocol. Consensus statement of the Study Group of Sport Cardiology of the Working Group of Cardiac Rehabilitation and Exercise Physiology and the Working Group of Myocardial and Pericardial Diseases of the European Society of Cardiology. Eur Heart J 2005;26:516-24.

12. Corrado D, Basso C, Pavei A, et al. Trends in sudden cardiovascular death in young competitive athletes after implementation of a preparticipation screening program. JAMA 2006;296:1593-601.

Affiliations: Department of Medicine, Division of Cardiology (Moayedi, Kumareswaran, Dorian), University of Toronto, Toronto, Ont.; Division of Cardiology (Kumareswaran, Dorian), St. Michael's Hospital, Toronto, Ont.

Contributors: All three authors contributed to the conception, preparation and editing of the manuscript; approved the version to be published; and agree to act as guarantors of the work.

Acknowledgements: The authors would like to thank Dr. Paul Angaran and Dr. Iqwal Mangat for valuable comments on and edits to the manuscript. 